\title{
A New Framework for Consensus for Discrete-Time Directed Networks of Multi-agents with Distributed Delays
}

\author{
Yurong Liu, Daniel W. C. Ho and Zidong Wang
}

\begin{abstract}
In this paper, the distributed consensus problem is considered for discrete-time delayed networks of dynamic agents with fixed topologies, where the networks under investigation are directed and the time-delays involved are distributed time delays including a single or multiple time delay(s) as special cases. By using the invariance principle of delay difference systems, a new unified framework is established to deal with the consensus for the discrete-time delayed multi-agent system. It is shown that the addressed discrete-time network with arbitrary distributed time delays reaches consensus provided that it is strongly connected. A numerical example is presented to illustrate the proposed methods.
\end{abstract}

\section{Keywords}

Consensus; Discrete-time multi-agent network; Directed network; Distributed time delay; Invariance principle.

\section{INTRODUCTION}

Coordination phenomena are ubiquitous in the natural world such as birds flocking and fish schooling [21]. Roughly speaking, the coordination phenomenon of networked multi-agent systems is characterized by the fact that each agent adjusts its own state by locally coupling with its neighbors (i.e. via the information from its neighbors) to achieve a common collective objective. In such a case, some natural questions arise as follows: 1) how can local communications and cooperations among individuals lead to certain desirable global behaviors? 2) what are the underlying mechanisms behind the coordination phenomena? and 3) which characters have significant influences on the coordination of the networked multi-agent systems? To answer these questions, various models and algorithm have been proposed and analyzed in the literature. Recently, the coordination problems of networked multi-agent systems have been attracting considerable research interests and a large number of results have been reported, see e.g. [13, 14,22,28].

In many practical applications, the study of coordination problems has been motivated by different realworld phenomena involving information flow among agents such as flocking, swarming, synchronization, distributed decision making and schooling; see e.g. [29] for a survey. In particular, consensus problems for networked dynamic systems have been extensively dealt with in the past few years $[8,17,18,23-27,36]$. Among

This work was supported in part by City University of Hong Kong under Grant 7008114, the Royal Society of the UK, the National Natural Science Foundation of China under Grants 60774073 and 61074129, and the Natural Science Foundation of Jiangsu Province of China under Grant BK2010313.

Y. Liu is with the Department of Mathematics, Yangzhou University, Yangzhou 225002, China.

D. W. C. Ho is with the Department of Mathematics, City University of Hong Kong, Hong Kong.

Z. Wang is with the Department of Information Systems and Computing, Brunel University, Uxbridge, Middlesex, UB8 3PH, United Kingdom. (Email: Zidong.Wang@brunel.ac.uk)

Email: liuyurong@gmail.com (Y. Liu), madaniel@cityu.edu.hk (D. W. C. Ho), Zidong.Wang@brunel.ac.uk (Z. Wang) 
others, the algebraic graph theory [7] appears to be one of the main tools used to analyze the consensus problem; see $[8,11,24,25,27]$. By using the graph theory, each agent is modeled as a vertex of a graph, and an edge of the graph joins node $i$ to node $j$ if agent $j$ is receiving information from agent $i$. Conditions for asymptotic consensus under a variety of assumptions on interagent communication have been recently published [30,33]. In [33], a simple discrete-time model has been proposed to simulate a group of autonomous agents moving in the plane with the same speed but different headings. The model addressed in [33] is essentially a simplified model introduced earlier by Reynolds in [30]. It has been shown that the network connectivity is a key factor in reaching consensus $[3,11,24,27]$. It has also been proven that the consensus in a network with a dynamically changing topology can be reached if and only if the time-varying network topology contains a spanning tree frequently enough as the network evolves with time [11,27]. Recently, stochastic approximation-type algorithms with a decreasing step size have been developed, and almost sure convergence has been established for consensus seeking; see e.g. [10] and the references therein.

Time-delay is well known to be an inherent feature of signal transmission over networks in practical applications, and is also recognized as one of the main sources for causing instability and poor performances of systems [1]. Here we shall discuss some of the recent work on consensus problems for delayed multi-agent networks. In [24], communication time-delays was first taken into account for continuous-time undirected networks with fixed topology and, based on the Laplace transform the consensus problem was analyzed with the time-delays in all channels equal to a common constant. In [19], a sufficient condition was derived to ensure the networks with the time-dependent communication patterns and a common time-delay in communication between distinct agents to reach consensus asymptotically. These results were further extended to the continuous-time directed networks with non-uniform delays [17].

Recently, the consensus problem in discrete-time multi-agent systems with time-delay has begun to attract the attention from researchers. For instance, [32] studied the state synchronization for systems with fixed undirected topology and time-delay, and based on the properties of non-negative matrices it was shown that the all agent states in the system converge to a single value regardless of the size of communication delays. In [5], asynchronous protocols involving time-delay were proposed, and the resulting consensus problem was investigated as well by means of graph theory, matrix theory and asynchronous theory. Also in [39], the model of networks of dynamic agents was extended to the case with multiple time-delays and it was proved that if the communication topology, time-delays, and weighting factors are time-invariant, then the necessary and sufficient condition that the multi-agent system solves a consensus problem is that the communication topology, represented by a directed graph, has spanning trees. Very recently, in [35], an new approach, i.e., pre-leader-follower decomposition was introduced to deal with the consensus problem for the discrete time multi-agent systems with fixed topology and time delays. By augmenting the state vector of the system, the consensus-seeking for the system with time delays reduced to the consensus problem for a system without time delays. In another paper [31], the linear matrix inequality approach was also used to investigate consensus problems in undirected networks of dynamic agents with fixed and switching topologies as well as multiple time-varying communication delays.

It should be noted that, in spite of much attention paid to the consensus problem for discrete-time delayed systems, the investigation has been made mostly on the systems including either a single or multiple time delay(s). On the other hand, another time-delay, namely infinite distributed time delay (simply called distributed time delay) has been also introduced to describe the dependence of the future state of a system on 
its past history. Multi-agent network usually ha a spatial nature due to the presence of an amount of parallel pathways of a variety of node sizes and lengths. Therefore, a great deal of research effort has been made on both theory and applications for such distributedly delayed systems, such as dynamical analysis, asymptotic behavior, and control and synchronization $[2,12,16,34,37,38]$. Up to date, however, to the best of the authors' knowledge, the consensus problems for discrete-time directed multi-agent systems with distributed time delays have received little research attention due mainly to the mathematical difficulties. In particular, since such systems cannot be expressed in the form of matrices, the commonly used approaches such as matrix analysis, algebraic connectivity and pre-leader-follower decomposition are no longer directly applicable to deal with the consensus problem. It is, therefore, our intention in this paper to tackle such an important yet challenging problem.

In this paper, we deal with the distributed consensus problem for the discrete-time directed multi-agent systems with distributed time delays. Under the assumption that the multi-agent network is strongly connected, we prove that the delayed discrete-time network reaches consensus, and a simulation example is exploited to illustrate the derived theory. The contribution of the paper is threefold:

(1) for the consensus seeking, the distributed time delays are first taken into account in the discrete-time directed multi-agent systems, and a single or multiple time delay(s) can be viewed as the special cases;

(2) different from most of the existing results, we develop a new unified framework to cope with the consensus for the delayed discrete-time agent systems by a blend of matrix theory, spectral graph theory, and especially a discrete-time version of LaSalle's invariance principle, which may be of independent interest. It is worth pointing out that our main results are also valid for the case of a single or multiple time delay(s); and

(3) some new techniques are employed in this paper. In particular, a key inequality and an appropriate Lyapunov-Krasovskii functional will be introduced to handle the distributed time delays, and they play a crucial role in the derivation of our main results.

The paper is organized as follows. In Section II, we introduce some basic concepts and formally state the problem. Section III contain our main results and proofs. Section IV provides an illustrative example. Finally, Section V concludes the paper with a summary of our results.

Notation The notation used here is fairly standard except where otherwise stated. Throughout this paper, $\mathbb{N}, \mathbb{Z}, \mathbb{Z}_{+}$and $\mathbb{Z}_{-}$stand for, respectively, the set of natural numbers, the set of integers, the set of non-negative integers and the set of non-positive integers. $\mathbb{R}, \mathbb{R}^{n}$ and $\mathbb{R}^{n \times m}$ denote, respectively, the set of real numbers, the $n$ dimensional Euclidean space and the set of all $n \times m$ real matrices. The superscript $T$ represents the transpose for a matrix, and $|\cdot|$ may stand for any absolute value of real numbers or the standard Euclidean norm from the context.

\section{Problem Formulation}

Consider $n$ agents distributed according to a directed graph $\mathcal{G}=(\mathcal{V}, \mathcal{E})$ with a set of nodes $\mathcal{V}=\{1,2, \ldots, n\}$, a set of edges $\mathcal{E} \in \mathcal{V} \times \mathcal{V}$, and a weighted adjacency matrix $A=\left[a_{i j}\right]$ with nonnegative adjacency elements $a_{i j}$. In $\mathcal{G}$, the $i$ th node represents $i$ th agent, and a directed edge (simply called an edge) from node $i$ to node $j$ denoted as an ordered pair $(i, j) \in \mathcal{E}$ represents a unidirectional information exchanges link from node $i$ to node $j$, that is, agent $j$ can receive or obtain information from agent $i$, but not necessarily vice versa. We assume that the graph has no self-loops, namely there is no edge between a node and itself, or $(i, i) \notin \mathcal{E}$. The set of neighbors of node $i$ is denoted by $\mathcal{N}_{i}=\{j:(j, i) \in \mathcal{E}\}$. A weighted adjacency matrix $A=\left[a_{i j}\right] \in \mathbb{R}^{n} \times \mathbb{R}^{n}$, associated with a weighted directed graph, is defined such that $a_{i j}$ is a positive weight if only $(j, i) \in \mathcal{E}$ (since 
the graph has no self-loops, it is obvious that $a_{i i}=0$, for all $i \in \mathcal{V}$ ). In other words, $a_{i j}>0$, if $j \in \mathcal{N}_{i}$, otherwise $a_{i j}=0$. A directed path (simply called a path) of length $k$ from $v_{t}$ to $v_{l}(t, l \in \mathcal{V}$ ) is a sequence of edges $\left(i_{0}, i_{1}\right),\left(i_{1}, i_{2}\right), \ldots,\left(i_{k}, i_{k+1}\right)$ with $i_{0}=t, i_{k+1}=l$ and $\left(i_{s}, i_{s+1}\right) \in \mathcal{E}$ for $s=0,1, \ldots, k$. A graph $\mathcal{G}$ is said to be strongly connected if there exists a path between any two distinct nodes in it. Throughout this paper, the two names, agent and node, will be used interchangeably.

Now let us investigate the dynamics of $n$ agents distributed over a directed graph $\mathcal{G}$. Let $x_{i}(k) \in \mathbb{R}$ denote the state of node $i$ at time $k, \mathbf{x}(k)=\left[x_{1}(k), x_{2}(k), \ldots, x_{n}(k)\right]^{T}$ be the state of the system accordingly, and $A=\left[a_{i j}\right]$ be the weighted adjacency matrix associated with the graph. The dynamics of discrete-time multiagent network under consideration is governed by:

$$
x_{i}(k+1)=x_{i}(k)+\sum_{j \in \mathcal{N}_{i}} a_{i j}\left(\sum_{v=0}^{+\infty} c_{i j v} x_{j}(k-v)-x_{i}(k)\right), \quad i \in \mathcal{V},
$$

where $a_{i j} \geq 0$ describes the communication link from node $j$ to node $i$, while the weight coefficient $c_{i j v} \geq 0$, and is subject to the following assumption.

\section{Assumption 1:}

$$
\sum_{v=0}^{+\infty} c_{i j v}=1, \quad j \in \mathcal{N}_{i} .
$$

Notice that the term $\sum_{v=0}^{+\infty} c_{i j v} x_{j}(k-v)-x_{i}(k)$ in Eq. (1) can be rewritten as $\sum_{v=0}^{+\infty} c_{i j v}\left[x_{j}(k-v)-x_{i}(k)\right]$, and the weight coefficient $c_{i j v}$ represents the strength of influence on node $i$ from the past history of node $j$. In Eq. (1), $c_{i j v}$ is defined only for $j \in \mathcal{N}_{i}$. For convenience, we add the definition of $c_{i j v}$ for $j \notin \mathcal{N}_{i}$ by letting

$$
c_{i j v}= \begin{cases}1, & \text { for } v=0 \\ 0, & v \geq 1\end{cases}
$$

Accordingly, for all $i, j \in \mathcal{V}$, the following holds:

$$
\sum_{v=0}^{+\infty} c_{i j v}=1
$$

Remark 1: The multi-agent system (1) is rather general, and some well-known systems may be viewed as its special cases. For example, letting $\tau_{i j} \in \mathbb{Z}^{+}$, and letting $c_{i j v}=0$ for all $v>\tau_{i j}$, then the system (1) reduces to

$$
x_{i}(k+1)=x_{i}(k)+\sum_{j \in \mathcal{N}_{i}} a_{i j}\left(\sum_{v=0}^{\tau_{i j}} c_{i j v} x_{j}(k-v)-x_{i}(k)\right), \quad i \in \mathcal{V} .
$$

In addition, letting $c_{i j v}=1$ for $v=\tau_{i j}$, and $c_{i j v}=0$ otherwise, the system (1) is further simplified as

$$
x_{i}(k+1)=x_{i}(k)+\sum_{j \in \mathcal{N}_{i}} a_{i j}\left(x_{j}\left(k-\tau_{i j}\right)-x_{i}(k)\right), \quad i \in \mathcal{V}
$$

Note that the consensus problem for systems (3) and (4) have been investigated in the literature; see e.g. [5, $25,31,35,39]$ and the references therein.

For the interaction topology of multi-agent system (1), we make the following assumption.

Assumption 2: The graph $\mathcal{G}=(\mathcal{V}, \mathcal{E})$ is strongly connected.

Let $B=\left[b_{i j}\right]$ with $b_{i j}=a_{i j}$ for $i \neq j$ and $b_{i i}=1-\sum_{j \neq i} a_{i j}$. Then, the system (1) can be rewritten as

$$
x_{i}(k+1)=b_{i i} x_{i}(k)+\sum_{j \neq i} b_{i j} \sum_{v=0}^{+\infty} c_{i j v} x_{j}(k-v), \quad i \in \mathcal{V} .
$$


In view of the definitions of $b_{i j}(i \neq j)$ and $b_{i i}$, it follows that

$$
\sum_{j=1}^{n} b_{i j}=1, \text { for all } i \text {. }
$$

Remark 2: Since $i \notin \mathcal{N}_{i}, a_{i i}=0$, and $b_{i j}=a_{i j}=0$ for $j \notin \mathcal{N}_{i}$ and $j \neq i$, it is clear that Eq. (5) is equivalent to Eq. (1).

Assumption 3: $b_{i i}>0$ for any $i \in \mathcal{V}$.

Definition 1: The multi-agent network (5) is said to reach consensus if, for any solution $\mathbf{x}(k)=\left(x_{1}(k), x_{2}(k)\right.$, $\left.\ldots, x_{n}(k)\right)^{T}$ of system $(5)$, there exists a real number $a^{*}$ such that $\lim _{k \rightarrow \infty} x_{i}(k)=a^{*}$ holds for all $i \in \mathcal{V}$.

In this paper, we aim to investigate the consensus problem for discrete-time system with arbitrary distributed time delays. By constructing a suitable Lyapunov-Krasovskii functional and employing invariance principle for delayed difference systems. We shall prove that network (5) reaches consensus under assumption of the strong connectedness.

\section{Main Results And Proofs}

This section is devoted to the consensus analysis for system (5). To start with, let us introduce two lemmas to be used in deriving our results.

Lemma 1 ( [9]) Let $A=\left[a_{i j}\right] \in \mathbb{R}^{n \times n}$ is nonnegative matrix, i.e., $a_{i j} \geq 0$, and let $\rho(A)$ be the spectral radius (called the Perron root of $A$ ). Suppose that $A$ is strongly connected, then there is a positive vector $x$ such that $A x=\rho(A) x$.

Lemma $2([15,16])$ Let $M \in \mathbb{R}^{n \times n}$ be a positive semi-definite matrix, $\mathbf{x}_{i} \in \mathbb{R}^{n}$, and scalar constant $a_{i} \geq 0$ for $i \in \mathbb{N}$. If the series concerned is convergent, then the following inequality holds:

$$
\left(\sum_{i=1}^{+\infty} a_{i} \mathbf{x}_{i}\right)^{T} M\left(\sum_{i=1}^{+\infty} a_{i} \mathbf{x}_{i}\right) \leq\left(\sum_{i=1}^{+\infty} a_{i}\right) \sum_{i=1}^{+\infty} a_{i} \mathbf{x}_{i}^{T} M \mathbf{x}_{i} .
$$

Let $\boldsymbol{X}=\left\{\phi: \mathbb{Z}_{-} \rightarrow \mathbb{R}^{n}\right.$ and $\phi$ is bounded $\}$ with the norm defined by $\|\phi\|=\max \left\{|\phi(s)|: s \in \mathbb{Z}_{-}\right\}$. For a sequence $\left\{\mathbf{x}(k) \in \mathbb{R}^{n}, k \in \mathbb{Z}\right\}$, define $\mathbf{x}_{k} \in \boldsymbol{X}$ by $\mathbf{x}_{k}(s)=\mathbf{x}(k+s)$ for $s \in \mathbb{Z}_{-}$. Then, for any given initial value $\phi \in \boldsymbol{X}$, there is a solution of the system (5) through $(0, \phi)$, which is denoted as $\mathbf{x}(\phi)(\cdot)$, or simply $\mathbf{x}(\cdot)$. Then, with these symbols, it is obvious that $\mathbf{x}_{0}=\phi$ if $\{\mathbf{x}(k)\}$ is a solution of network (5) with initial value $\phi$.

The main result of this paper is given as follows.

Theorem 1: Under Assumptions 1 and 3, the discrete-time multi-agent network (5) with arbitrary distributed time delays reaches consensus.

For analysis of the consensus problems of network (5), there are two difficult issues arisen as follows: (i) how to handle the distributed time delays in the given network; (ii) how to handle the non-uniqueness of equilibrium points of network (5). To deal with these problems, the construction of a Lyapunov-Krasovskii functional $V$ is quite crucial, and is introduced from (9) to be given later. Here, the proof consists of three main steps. Step 1 is to construct a candidate Lyapunov-Krasovskii functional $V\left(\mathbf{x}_{k}\right)$, and verify the result of $\Delta V\left(\mathbf{x}_{k}\right) \leq 0$; Step 2 is to characterize the maximal positively invariant set [41] relative to (5); and Step 3 is devoted to the proof of the consensus.

\section{Proof of Theorem 1}


Step 1: First of all, by Lemma 1, there exists a positive left eigenvector $\xi=\left[\xi_{1}, \xi_{2}, \ldots, \xi_{n}\right]^{T}$ of $B$ such that

$$
\xi^{T} B=\xi^{T}, \quad \text { and } \quad \sum_{i=1}^{n} \xi_{i}=1
$$

In order to deal with the consensus problem, we construct the following Lyapunov-Krasovskii functional:

$$
V\left(\mathbf{x}_{k}\right)=V_{1}\left(\mathbf{x}_{k}\right)+V_{2}\left(\mathbf{x}_{k}\right),
$$

where

$$
V_{1}\left(\mathbf{x}_{k}\right)=\sum_{i=1}^{n} \xi_{i} x_{i}^{2}(k)
$$

and

$$
V_{2}\left(\mathbf{x}_{k}\right)=\sum_{i=1}^{n} \xi_{i} \sum_{j=1}^{n} \sum_{l=1}^{n} b_{i j} b_{i l} V_{i j l}\left(\mathbf{x}_{k}\right)
$$

with

$$
V_{i j l}\left(\mathbf{x}_{k}\right)=\frac{1}{2}\left[\sum_{v=0}^{+\infty} c_{i j v} \sum_{s=k-v}^{k-1} x_{j}^{2}(s)+\sum_{v=0}^{+\infty} c_{i l v} \sum_{s=k-v}^{k-1} x_{l}^{2}(s)\right], \text { for } 1 \leq j, l \leq n .
$$

Noticing that for convenience in a unified frame to deal with the different cases of time delays, throughout our analysis, for $a_{k} \in \mathbb{R}$, we adopt the convention:

$$
\sum_{k=s}^{t} a_{k}=0, \text { if } t<s
$$

From the convention and definition of $V_{i j l}$, it is obvious that

$$
V_{i i i}\left(\mathbf{x}_{k}\right)=0, V_{i j i}\left(\mathbf{x}_{k}\right)=\frac{1}{2} \sum_{v=0}^{+\infty} c_{i j v} \sum_{s=k-v}^{k-1} x_{j}^{2}(s), V_{i i l}\left(\mathbf{x}_{k}\right)=\frac{1}{2} \sum_{v=0}^{+\infty} c_{i l v} \sum_{s=k-v}^{k-1} x_{l}^{2}(s) .
$$

And it is also clear that $V_{i j l}=V_{i l j}$.

Now, the difference of $V\left(\mathbf{x}_{k}\right)$ along the solutions of network (5) is defined to be

$$
\Delta V\left(\mathbf{x}_{k}\right)=\Delta V_{1}\left(\mathbf{x}_{k}\right)+\Delta V_{2}\left(\mathbf{x}_{k}\right)
$$

Here,

$$
\begin{aligned}
\Delta V_{1}\left(\mathbf{x}_{k}\right)= & V_{1}\left(\mathbf{x}_{k+1}\right)-V_{1}\left(\mathbf{x}_{k}\right) \\
= & \sum_{i=1}^{n} \xi_{i} x_{i}^{2}(k+1)-\sum_{i=1}^{n} \xi_{i} x_{i}^{2}(k) \\
= & \sum_{i=1}^{n} \xi_{i}\left[b_{i i} x_{i}(k)+\sum_{j \neq i} b_{i j} \sum_{v=0}^{+\infty} c_{i j v} x_{j}(k-v)\right]^{2}-\sum_{i=1}^{n} \xi_{i} x_{i}^{2}(k) \\
= & \sum_{i=1}^{n} \xi_{i}\left[b_{i i}^{2} x_{i}^{2}(k)+\sum_{j \neq i} b_{i j}^{2}\left(\sum_{v=0}^{+\infty} c_{i j v} x_{j}(k-v)\right)^{2}+2 b_{i i} x_{i}(k) \sum_{j \neq i} b_{i j}\left(\sum_{v=0}^{+\infty} c_{i j v} x_{j}(k-v)\right)\right. \\
& \left.+2 \sum_{j \neq i} \sum_{\substack{l>j \\
l \neq i}} b_{i j} b_{i l}\left(\sum_{v=0}^{+\infty} c_{i j v} x_{j}(k-v)\right)\left(\sum_{v=0}^{+\infty} c_{i j v} x_{l}(k-v)\right)\right]-\sum_{i=1}^{n} \xi_{i} x_{i}^{2}(k),
\end{aligned}
$$


and

$$
\begin{aligned}
\Delta V_{2}\left(\mathbf{x}_{k}\right)= & V_{2}\left(\mathbf{x}_{k+1}\right)-V_{2}\left(\mathbf{x}_{k}\right) \\
= & \sum_{i=1}^{n} \xi_{i} \sum_{j=1}^{n} \sum_{l=1}^{n} b_{i j} b_{i l} V_{i j l}\left(\mathbf{x}_{k+1}\right)-\sum_{i=1}^{n} \xi_{i} \sum_{j=1}^{n} \sum_{l=1}^{n} b_{i j} b_{i l} V_{i j l}\left(\mathbf{x}_{k}\right) \\
= & \sum_{i=1}^{n} \xi_{i} \sum_{j=1}^{n} \sum_{l=1}^{n} b_{i j} b_{i l} \Delta V_{i j l}\left(\mathbf{x}_{k}\right) \\
= & \sum_{i=1}^{n} \xi_{i}\left[\sum_{j=1}^{n} \sum_{l=j} b_{i j} b_{i l} \Delta V_{i j l}\left(\mathbf{x}_{k}\right)+\sum_{j=1}^{n} \sum_{l \neq j} b_{i j} b_{i l} \Delta V_{i j l}\left(\mathbf{x}_{k}\right)\right] \\
= & \sum_{i=1}^{n} \xi_{i}\left[b_{i i}^{2} \Delta V_{i i i}\left(\mathbf{x}_{k}\right)+\sum_{j \neq i} b_{i j}^{2} \Delta V_{i j j}\left(\mathbf{x}_{k}\right)+2\left(\sum_{j \neq i} b_{i i} b_{i j} \Delta V_{i j i}\left(\mathbf{x}_{k}\right)\right.\right. \\
& \left.\left.+\sum_{j \neq i} \sum_{\substack{l>j \\
l \neq i}} b_{i j} b_{i l} \Delta V_{i j l}\left(\mathbf{x}_{k}\right)\right)\right]
\end{aligned}
$$

where $\Delta V_{i j l}\left(\mathbf{x}_{k}\right)=V_{i j l}\left(\mathbf{x}_{k+1}\right)-V_{i j l}\left(\mathbf{x}_{k}\right)$.

By the definition of $V_{i j l}\left(\mathbf{x}_{k}\right)$, it follows that

$$
\begin{aligned}
\Delta V_{i j l}\left(\mathbf{x}_{k}\right)= & \frac{1}{2}\left[\sum_{v=0}^{+\infty} c_{i j v} \sum_{s=k+1-v}^{k} x_{j}^{2}(s)+\sum_{v=0}^{+\infty} c_{i l v} \sum_{s=k+1-v}^{k} x_{l}^{2}(s)\right] \\
& -\frac{1}{2}\left[\sum_{v=0}^{+\infty} c_{i j v} \sum_{s=k-v}^{k-1} x_{j}^{2}(s)+\sum_{v=0}^{+\infty} c_{i l v} \sum_{s=k-v}^{k-1} x_{l}^{2}(s)\right] \\
= & \frac{1}{2}\left[\sum_{v=0}^{+\infty} c_{i j v}\left(x_{j}^{2}(k)-x_{j}^{2}(k-v)\right)+\sum_{v=0}^{+\infty} c_{i l v}\left(x_{l}^{2}(k)-x_{l}^{2}(k-v)\right)\right] \\
= & \frac{1}{2}\left[x_{j}^{2}(k)-\sum_{v=0}^{+\infty} c_{i j v} x_{j}^{2}(k-v)+x_{l}^{2}(k)-\sum_{v=0}^{+\infty} c_{i l v} x_{l}^{2}(k-v)\right] .
\end{aligned}
$$

Notice that we have used $\sum_{v=0}^{+\infty} c_{i j v}=1$, and $\sum_{v=0}^{+\infty} c_{i l v}=1$.

Substituting (17) into (16) yields that

$$
\begin{aligned}
\Delta V_{2}\left(\mathbf{x}_{k}\right)= & \sum_{i=1}^{n} \xi_{i}\left[\sum_{j \neq i} b_{i j}^{2}\left(x_{j}^{2}(k)-\sum_{v=0}^{+\infty} c_{i j v} x_{j}^{2}(k-v)\right)+\sum_{j \neq i} b_{i i} b_{i j}\left(x_{j}^{2}(k)-\sum_{v=0}^{+\infty} c_{i j v} x_{j}^{2}(k-v)\right)\right. \\
& \left.+\sum_{\substack { j \neq i \\
\begin{subarray}{c}{l>j \\
l \neq i{ j \neq i \\
\begin{subarray} { c } { l > j \\
l \neq i } }\end{subarray}} b_{i j} b_{i l}\left(x_{j}^{2}(k)-\sum_{v=0}^{+\infty} c_{i j v} x_{j}^{2}(k-v)+x_{l}^{2}(k)-\sum_{v=0}^{+\infty} c_{i l v} x_{l}^{2}(k-v)\right)\right],
\end{aligned}
$$

From Lemma 2, it follows that

$$
\left(\sum_{v=0}^{+\infty} c_{i j v} x_{j}(k-v)\right)^{2} \leq\left(\sum_{v=0}^{+\infty} c_{i j v}\right) \sum_{v=0}^{+\infty} c_{i j v} x_{j}^{2}(k-v)=\sum_{v=0}^{+\infty} c_{i j v} x_{j}^{2}(k-v) .
$$


Thus, (18), together with (19), implies that

$$
\begin{aligned}
\Delta V_{2}\left(\mathbf{x}_{k}\right) \leq & \sum_{i=1}^{n} \xi_{i}\left[\sum_{j \neq i} b_{i j}^{2}\left(x_{j}^{2}(k)-\left(\sum_{v=0}^{+\infty} c_{i j v} x_{j}(k-v)\right)^{2}\right)+\sum_{j \neq i} b_{i i} b_{i j}\left(x_{j}^{2}(k)-\left(\sum_{v=0}^{+\infty} c_{i j v} x_{j}(k-v)\right)^{2}\right)\right. \\
& \left.+\sum_{j \neq i} \sum_{\substack{l>j \\
l \neq i}} b_{i j} b_{i l}\left(x_{j}^{2}(k)-\left(\sum_{v=0}^{+\infty} c_{i j v} x_{j}(k-v)\right)^{2}+x_{l}^{2}(k)-\left(\sum_{v=0}^{+\infty} c_{i l v} x_{l}(k-v)\right)^{2}\right)\right] .
\end{aligned}
$$

Substituting (15) and (20) into (14) results in

$$
\begin{aligned}
\Delta V\left(\mathbf{x}_{k}\right) \leq & \sum_{i=1}^{n} \xi_{i}\left[b_{i i}^{2} x_{i}^{2}(k)+\sum_{j \neq i} b_{i j}^{2} x_{j}^{2}(k)+\sum_{j \neq i} b_{i i} b_{i j}\left(x_{j}^{2}(k)-\left(\sum_{v=0}^{+\infty} c_{i j v} x_{j}(k-v)\right)^{2}\right.\right. \\
& \left.+2 x_{i}(k) \sum_{v=0}^{+\infty} c_{i j v} x_{j}(k-v)\right)+\sum_{j \neq i} \sum_{\substack{l>j \\
l \neq i}} b_{i j} b_{i l}\left(x_{j}^{2}(k)-\left(\sum_{v=0}^{+\infty} c_{i j v} x_{j}(k-v)^{2}+x_{l}^{2}(k)\right.\right. \\
& \left.\left.-\left(\sum_{v=0}^{+\infty} c_{i l v} x_{l}(k-v)\right)^{2}+2\left(\sum_{v=0}^{+\infty} c_{i j v} x_{j}(k-v)\right)\left(\sum_{v=0}^{+\infty} c_{i j v} x_{l}(k-v)\right)\right)\right]-\sum_{i=1}^{n} \xi_{i} x_{i}^{2}(k) \\
= & \sum_{i=1}^{n} \xi_{i}\left[\sum_{j=1}^{n} b_{i j}^{2} x_{j}^{2}(k)+\sum_{j \neq i} b_{i i} b_{i j}\left(x_{i}^{2}(k)+x_{j}^{2}(k)-\left(x_{i}(k)-\sum_{v=0}^{+\infty} c_{i j v} x_{j}(k-v)^{2}\right)\right.\right. \\
& \left.+\sum_{j \neq i} \sum_{\substack{l>j \\
l \neq i}} b_{i j} b_{i l}\left(x_{j}^{2}(k)+x_{l}^{2}(k)-\left(\sum_{v=0}^{+\infty} c_{i j v} x_{j}(k-v)-\sum_{v=0}^{+\infty} c_{i j v} x_{l}(k-v)\right)^{2}\right)\right] \\
& -\sum_{i=1}^{n} \xi_{i} x_{i}^{2}(k) \\
= & \sum_{i=1}^{n} \xi_{i}\left[\sum_{j=1}^{n} b_{i j}^{2} x_{j}^{2}(k)+\sum_{j=1}^{n} \sum_{l>j} b_{i j} b_{i l}\left(x_{j}^{2}(k)+x_{l}^{2}(k)\right)\right]-\sum_{i=1}^{n} \xi_{i} x_{i}^{2}(k) \\
& -\sum_{i=1}^{n} \xi_{i}\left[\sum_{j \neq i} b_{i i} b_{i j}\left(x_{i}(k)-\sum_{v=0}^{+\infty} c_{i j v} x_{j}(k-v)\right)^{2}\right. \\
& \left.+\sum_{j \neq i} \sum_{\substack{l>j \\
l \neq i}} b_{i j} b_{i l}\left(\sum_{v=0}^{+\infty} c_{i j v} x_{j}(k-v)-\sum_{v=0}^{+\infty} c_{i j v} x_{l}(k-v)\right)^{2}\right] . \\
&
\end{aligned}
$$


For the first term on the right hand side of (21), one has

$$
\begin{aligned}
& \sum_{i=1}^{n} \xi_{i}\left[\sum_{j=1}^{n} b_{i j}^{2} x_{j}^{2}(k)+\sum_{j=1}^{n} \sum_{l>j} b_{i j} b_{i l}\left(x_{j}^{2}(k)+x_{l}^{2}(k)\right)\right] \\
= & \sum_{i=1}^{n} \xi_{i}\left[\sum_{j=1}^{n} b_{i j}^{2} x_{j}^{2}(k)+\sum_{j=1}^{n} \sum_{l>j} b_{i j} b_{i l} x_{j}^{2}(k)+\sum_{j=1}^{n} \sum_{l>j} b_{i j} b_{i l} x_{l}^{2}(k)\right] \\
= & \sum_{i=1}^{n} \xi_{i}\left[\sum_{j=1}^{n} b_{i j}^{2} x_{j}^{2}(k)+\sum_{j=1}^{n} \sum_{l>j} b_{i j} b_{i l} x_{j}^{2}(k)+\sum_{l=1}^{n} \sum_{j>l} b_{i l} b_{i j} x_{j}^{2}(k)\right] \\
= & \sum_{i=1}^{n} \xi_{i}\left[\sum_{j=1}^{n} b_{i j}^{2} x_{j}^{2}(k)+\sum_{j=1}^{n} \sum_{l>j} b_{i j} b_{i l} x_{j}^{2}(k)+\sum_{j=1}^{n} \sum_{l<j} b_{i l} b_{i j} x_{j}^{2}(k)\right] \\
= & \sum_{i=1}^{n} \xi_{i}\left[\sum_{j=1}^{n} b_{i j}^{2} x_{j}^{2}(k)+\sum_{j=1}^{n} \sum_{l \neq j} b_{i j} b_{i l} x_{j}^{2}(k)\right] \\
= & \sum_{i=1}^{n} \xi_{i} \sum_{j=1}^{n} \sum_{l=1}^{n} b_{i j} b_{i l} x_{j}^{2}(k) \\
= & \sum_{i=1}^{n} \xi_{i} \sum_{j=1}^{n} b_{i j} x_{j}^{2}(k) \sum_{l=1}^{n} b_{i l} .
\end{aligned}
$$

It follows from $\sum_{j=1}^{n} b_{i j}=1(i \in \mathcal{V})$ and the equality (8) that

$$
\begin{aligned}
& \sum_{i=1}^{n} \xi_{i} \sum_{j=1}^{n} b_{i j} x_{j}^{2}(k) \sum_{l=1}^{n} b_{i l}=\sum_{i=1}^{n} \xi_{i} \sum_{j=1}^{n} b_{i j} x_{j}^{2}(k) \\
= & \sum_{j=1}^{n} \sum_{i=1}^{n} \xi_{i} b_{i j} x_{j}^{2}(k)=\sum_{j=1}^{n} x_{j}^{2}(k) \sum_{i=1}^{n} \xi_{i} b_{i j}=\sum_{j=1}^{n} \xi_{j} x_{j}^{2}(k) .
\end{aligned}
$$

Equation (21), together with (22) and (23) implies that

$$
\begin{aligned}
\Delta V\left(\mathbf{x}_{k}\right)= & -\sum_{i=1}^{n} \xi_{i}\left[\sum_{j \neq i} b_{i i} b_{i j}\left(x_{i}(k)-\sum_{v=0}^{+\infty} c_{i j v} x_{j}(k-v)\right)^{2}\right. \\
& \left.+\sum_{j \neq i} \sum_{\substack{l>j \\
l \neq i}} b_{i j} b_{i l}\left(\sum_{v=0}^{+\infty} c_{i j v} x_{j}(k-v)-\sum_{v=0}^{+\infty} c_{i j v} x_{l}(k-v)\right)^{2}\right]
\end{aligned}
$$

By Assumption 3 and the definition of $b_{i j}, b_{i j} \geq 0$ for all $i, j$ and, therefore, it follows that

$$
\Delta V\left(\mathbf{x}_{k}\right) \leq 0
$$

Step 2: Let $E=\{\phi \in \boldsymbol{X}: \Delta V(\phi)=0\}$, and $M \subset E$ denote the maximal positively invariant set, which is discussed in [41] for discrete-time systems, relative to (5). Let $\hat{M} \stackrel{\text { def }}{=}\{\phi \in \boldsymbol{X}: \exists a \in \mathbb{R}$ such that $\phi(s)=$ $a \mathbf{1}$, for $\left.s \in \mathbb{Z}_{-}\right\}$where $\mathbf{1} \in \mathbb{R}^{n}$ denotes the vector with all entries equal to one. In view of (2) and $\left(2^{\prime}\right)$, it is easy to verify that

$$
M \supset \hat{M}
$$

We shall prove that

$$
M=\hat{M}
$$


Now it suffices to prove that

$$
M \subset \hat{M}
$$

To this end, for any $\phi \in M$, let $\{\mathbf{x}(k), k \in \mathbb{Z}\}$ be the solution of (5) relative to the initial value $\phi$. Then, by the definition of $M$, one has

$$
\Delta V\left(\mathbf{x}_{k}\right)=0, \text { for } k \geq 0 .
$$

By (24) and with $\xi_{i}>0$, clearly (28) is equivalent to

$$
\sum_{j \neq i} b_{i i} b_{i j}\left(x_{i}(k)-\sum_{v=0}^{+\infty} c_{i j v} x_{j}(k-v)\right)^{2}=0, \quad \text { for all } k \geq 0 \text { and } i \in \mathcal{V}
$$

and

$$
\sum_{j \neq i} \sum_{\substack{l>j \\ l \neq i}} b_{i j} b_{i l}\left(\sum_{v=0}^{+\infty} c_{i j v} x_{j}(k-v)-\sum_{v=0}^{+\infty} c_{i l v} x_{l}(k-v)\right)^{2}=0, \quad \text { for all } k \geq 0 \text { and } i \in \mathcal{V}
$$

Notice that $b_{i i} \neq 0$, and $\mathcal{N}_{i} \neq \emptyset$ since the graph $\mathcal{G}(\mathcal{V}, \mathcal{E})$ is strongly connected, and obviously $b_{i j} \neq 0(j \neq$ $i$ ) if and only if $j \in \mathcal{N}_{i}$. Then from Eq. (29a), we have

$$
x_{i}(k)=\sum_{v=0}^{+\infty} c_{i j v} x_{j}(k-v), \quad j \in \mathcal{N}_{i}\left(\text { or when } b_{i j} \neq 0\right) .
$$

With a view to Eq. (6), substituting (30) into (5) yields

$$
x_{i}(k+1)=b_{i i} x_{i}(k)+\sum_{j \neq i} b_{i j} x_{i}(k)=x_{i}(k), \quad i \in \mathcal{V} ;
$$

Hence, (30) and (31) imply that if $j_{0} \in \mathcal{N}_{i_{0}}$, then

$$
x_{i_{0}}(k)=\sum_{v=0}^{+\infty} c_{i j_{0} v} x_{j_{0}}(k-v)=x_{j_{0}}(k) .
$$

Furthermore, according to the strong connectedness of the graph $\mathcal{G}(\mathcal{V}, \mathcal{E})$, we have

$$
x_{i}(k)=x_{j}(k), \text { for any } k \in \mathbb{N} \text {, and } i, j \in \mathcal{V} .
$$

Noticing that $M$ is positively invariant set relative to (5), and from (31) and (33), it is not difficult to see

$$
\mathbf{x}_{k} \in \hat{M}, \quad \mathbf{x}_{k+1}=\mathbf{x}_{k},
$$

Specially, letting $k=0$ in the first formula of (34), one has

$$
\phi=\mathbf{x}_{0} \in \hat{M} .
$$

Hence we arrive at $M \subset \hat{M}$, and $M=\hat{M}$ accordingly.

Step 3: Now we shall prove network (5) reaches the consensus. For this purpose, let $\{\mathbf{x}(k), k \in \mathbb{Z}\}$ be the solution of (5) relative to the initial value $\phi$. By the Invariance Principle for autonomous delay difference systems [41], there is a constant $c$ such that

$$
\mathbf{x}_{k} \rightarrow M \cap V^{-1}(c), \quad \text { as } k \rightarrow \infty,
$$


which means that there is a sequence $\left\{\phi^{(k)}\right\} \subset M$ with $\phi^{(k)}(s)=a_{k} \mathbf{1}$ for $s \in \mathbb{Z}_{\text {- such that }}$

$$
\lim _{n \rightarrow \infty}\left\|\mathbf{x}_{k}-\phi^{(k)}\right\|=0 \text { and } \lim _{n \rightarrow \infty} V\left(\mathbf{x}_{k}\right)=c
$$

From (37) and the continuity of $V(\cdot)$, it follows that

$$
\lim _{n \rightarrow \infty} V\left(\phi^{(k)}\right)=c .
$$

Through a straightforward calculation, we can deduce from (38) that there exists a real number $c^{*} \geq 0$, such that $\lim _{k \rightarrow \infty} a_{k}^{2}=c^{*}$, or

$$
\lim _{k \rightarrow \infty}\left|a_{k}\right|=\sqrt{c^{*}}
$$

However we can assert that

$$
\lim _{n \rightarrow \infty} a_{k}=a^{*}, \text { with } a^{*}=\sqrt{c^{*}} \text { or }-\sqrt{c^{*}} .
$$

In fact, from (39) it is obvious for $c^{*}=0$. Now consider the case that $c^{*}>0$. First, notice that since system (5) is autonomous its solutions are uniformly continuously dependent on the initial values. Therefore there exists a positive number $\delta$ such that for any two solutions $\{\mathbf{y}(k), k \in \mathbb{Z}\}$ and $\{\mathbf{z}(k), k \in \mathbb{Z}\}$ with initial values $\psi^{(1)}, \psi^{(2)} \in \boldsymbol{X}$, respectively, such that

$$
\left\|\mathbf{y}_{1}-\mathbf{z}_{1}\right\|<\sqrt{c^{*}} / 2, \quad \text { if }\left\|\psi^{(1)}-\psi^{(2)}\right\|<\delta
$$

According to (39), there is a positive integer $N_{2}$ such that

$$
|| a_{k}\left|-\sqrt{c^{*}}\right|<\sqrt{c^{*}} / 2, \text { when } k>N_{1} .
$$

Also by the first equality of (37), there exists a positive integer $N_{2}$ such that

$$
\left\|\mathbf{x}_{k}-\phi^{(k)}\right\|<\min \left\{\delta, \sqrt{c^{*}} / 2\right\}, \quad \text { when } k>N_{2} .
$$

Now assume that equality (40) is not true. Setting $N_{0}=\max \left\{N_{1}, N_{2}\right\}$, then we can deduce that there exist a positive integer $k_{0}>N_{0}$, such that $a_{k_{0}} a_{k_{0}+1}<0$. Without loss of generality, we assume that $a_{k_{0}}>0$ and $a_{k_{0}+1}<0$. Then it readily follows that

$$
\left\|\phi^{\left(k_{0}+1\right)}-\phi^{\left(k_{0}\right)}\right\|=\left(\left|a_{k_{0}+1}\right|+\left|a_{k_{0}}\right|\right) \sqrt{n} \geq\left|a_{k_{0}+1}\right|+\left|a_{k_{0}}\right|>\sqrt{c^{*}}
$$

where the last inequality follows from (42). On the other hand, noting that $\phi^{(k)}$ is fixed or invariant relative to (5), from (41) and (43) we have that

$$
\left\|\mathbf{x}_{k_{0}+1}-\phi^{\left(k_{0}\right)}\right\|<\sqrt{c^{*}} / 2
$$

which implies that

$$
\left\|\phi^{\left(k_{0}+1\right)}-\phi^{\left(k_{0}\right)}\right\| \leq\left\|\phi^{\left(k_{0}+1\right)}-\mathbf{x}_{k_{0}+1}\right\|+\left\|\mathbf{x}_{k_{0}+1}-\phi^{\left(k_{0}\right)}\right\|<\sqrt{c^{*}} / 2+\sqrt{c^{*}} / 2=\sqrt{c^{*}} .
$$

This is in contradiction with (44). Consequently (40) holds, and we further have

$$
\lim _{k \rightarrow \infty} \phi^{(k)}=\phi^{*}
$$


where $\phi^{*} \in \boldsymbol{X}$ with $\phi^{*}(s)=a^{*} \mathbf{1}$ for $s \in \mathbb{Z}_{-}$. Combination of (37) and (47) leads to

$$
\lim _{k \rightarrow \infty} \mathbf{x}_{k}=\phi^{*}
$$

which implies that

$$
\lim _{k \rightarrow \infty} \mathbf{x}(k)=a^{*} \mathbf{1}
$$

namely

$$
\lim _{k \rightarrow \infty} x_{i}(k)=a^{*}
$$

Therefore system (5) reaches consensus, and the proof of this theorem is complete.

Remark 3: The construction of $V_{1}\left(\mathbf{x}_{k}\right)$ is not trivial and the parameter $\xi$ plays a crucial role which can be seen in later derivation; on the other hand, $V_{2}\left(\mathbf{x}_{k}\right)$ is a necessary part for dealing with the distributed time delays.

Remark 4: Based on the particular choice of Lyapunov-Krasovskii functional $V\left(\mathbf{x}_{k}\right)$ in (9), it can be seen that all positive terms in $\Delta V_{1}\left(\mathbf{x}_{k}\right)$ and $\Delta V_{2}\left(\mathbf{x}_{k}\right)$ (see (15) and (20)) are canceled by those terms in $V_{1}\left(\mathbf{x}_{k}\right)$ (see (15) ). There Lemma 2 also plays a key role in handling $\Delta V_{2}\left(\mathbf{x}_{k}\right)$ and deriving (24). Note that (24) involves only a few negative terms, and the terms in square brackets are no longer related to the left eigenvector $\xi$ in (8). In addition, these terms are important and have been used in Step 2.

As mentioned earlier, the systems (3) and (4) are regarded as the special cases of system (5), and one therefore has

Corollary 1: Under Assumptions 2 and 3, the discrete-time multi-agent network (3) reaches consensus.

Corollary 2: Under Assumptions 2 and 3, the discrete-time multi-agent network (4) reaches consensus.

Remark 5: Corollaries (1) and (2) can be given a direct proof by making a slight modification to the proof of Theorem 1 with the help of another discrete-time version of LaSalle's invariance principle [40].

In this paper, we only consider scalar individual states, and it is easy to extend them to vector individual states. Consider the following multi-agent system of $n$-nodes with vector-valued states:

$$
x_{i}(k+1)=b_{i i} x_{i}(k)+\sum_{j \neq i} b_{i j} \sum_{v=0}^{+\infty} c_{i j v} x_{j}(k-v), \quad i \in \mathcal{V},
$$

where $x_{i}(k) \in \mathbb{R}^{m}$.

Assumption 4: Let $b_{i j} \geq 0, b_{i i}=1-\sum_{j \neq i}^{n} b_{i j}>0$ and $\tau_{i i}=0$. Assume that network (50) is strongly connected.

Theorem 2: Under Assumptions 1 and 4, the discrete-time multi-agent system (50) with arbitrary distributed time delays reaches consensus, i.e., for any given solution $x(k)=\left(x_{1}(k), x_{2}(k), \cdots, x_{n}(k)\right)^{T}$ of system (50), there exists a constant vector $x^{*} \in \mathbb{R}^{m}$ such that $\lim _{k \rightarrow \infty} x_{i}(k)=x^{*}, i \in \mathcal{V}$.

Proof: The proof of this theorem is similar to that of the previous theorem, and therefore it is omitted here.

\section{A NumericAl EXAMPle}

For the purpose of illustration, let us consider two examples. The first example is used to justify our assumption 3, and the second example to demonstrate the effectiveness of our main results. 
Example 1: Theorem 1 holds under Assumptions 1-3. We shall point out here that Assumption 3 is necessary for consensus reaching. This can be shown by the following example. For simplicity, we consider network (5) of two nodes given by:

$$
\left\{\begin{array}{l}
x_{1}(k+1)=x_{2}(k-1), \\
x_{2}(k+1)=x_{1}(k-1),
\end{array}\right.
$$

where the network is strongly connected, but $b_{11}=b_{22}=0$. Take an initial value $\phi$ with $\phi(-1)=\phi(0)=\left[\begin{array}{ll}1 & 2\end{array}\right]^{T}$, then the resulting solution $\mathbf{x}(k)$ relative to this initial value is given by

$$
\begin{cases}\mathbf{x}(k)=\left[\begin{array}{ll}
2 & 1
\end{array}\right]^{T}, & k=4 t+1,4 t+2, \text { for } t \in \mathbb{Z}_{+}, \\
\mathbf{x}(k)=\left[\begin{array}{ll}
1 & 2
\end{array}\right]^{T}, & k=4 t+3,4 t+4, \text { for } t \in \mathbb{Z}_{+} .\end{cases}
$$

Clearly, each individual state does not converges, and hence the network does not reach consensus.

Example 2: Consider the multi-agent network (5), and for simplicity, we take $n=6$. The interaction topology between the agents is shown in Fig. 1, and the other parameters are taken as follows:

$\left[b_{i j}\right]_{6 \times 6}=\left[\begin{array}{cccccc}0.6 & 0 & 0 & 0.4 & 0 & 0 \\ 0.2 & 0.8 & 0 & 0 & 0 & 0 \\ 0.4 & 0 & 0.6 & 0 & 0 & 0 \\ 0 & 0 & 0 & 0.5 & 0 & 0.5 \\ 0 & 0.4 & 0 & 0 & 0.6 & 0 \\ 0 & 0 & 0 & 0.2 & 0.4 & 0.4\end{array}\right] ; c_{12 v}=2^{-v}$ for $v \in \mathbb{Z}_{+} ; c_{21 v}= \begin{cases}1 / 10, & \text { if } v=0 ; \\ 2 / 5, & \text { if } v=1 ; \\ 3^{-v}, & \text { if } v \geq 2 ;\end{cases}$

$c_{31 v}=\left\{\begin{array}{ll}2 / 3, & \text { if } v=3 ; \\ 1 / 3, & \text { if } v=5 ; \\ 0, & \text { otherwise }\end{array} \quad c_{46 v}=\left\{\begin{array}{ll}3 / 5, & \text { if } v=2 ; \\ 2 / 5, & \text { if } v=4 ; \\ 0, & \text { otherwise }\end{array} \quad c_{52 v}= \begin{cases}3 / 5, & \text { if } v=3 ; \\ 1 / 5, & \text { if } v=5 ; \\ 1 / 5, & \text { if } v=6 ; \\ 0, & \text { otherwise; }\end{cases}\right.\right.$

$c_{63 v}=\left\{\begin{array}{ll}4 / 5, & \text { if } v=1 ; \\ 1 / 5, & \text { if } v=4 ; \\ 0, & \text { otherwise; }\end{array} \quad c_{65 v}= \begin{cases}1, & \text { if } v=5 \\ 0, & \text { otherwise }\end{cases}\right.$

Clearly, for the given parameters above, Assumptions 1-3 are satisfied, and from Theorem 1, the multi-agent system reaches consensus.

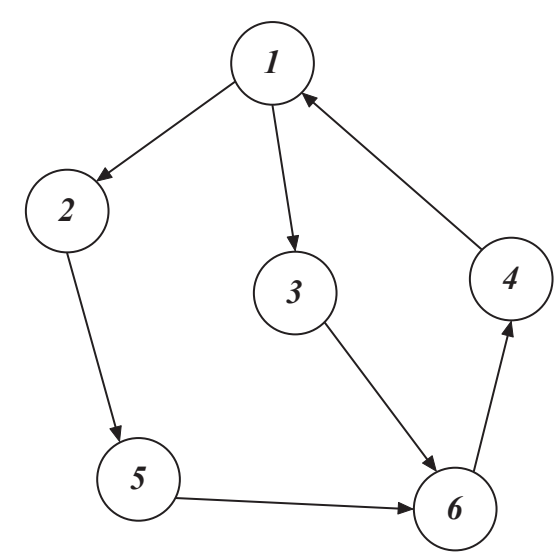

Fig. 1. The interaction topology of multi-agent system 
In Fig. 2, three sets of initial values are taken randomly to simulate the evolution of the states, and the numerical results show that for each initial value, the individual state of the multi-agent system converges to a constant limit, which agrees well with the proposed theoretical result.
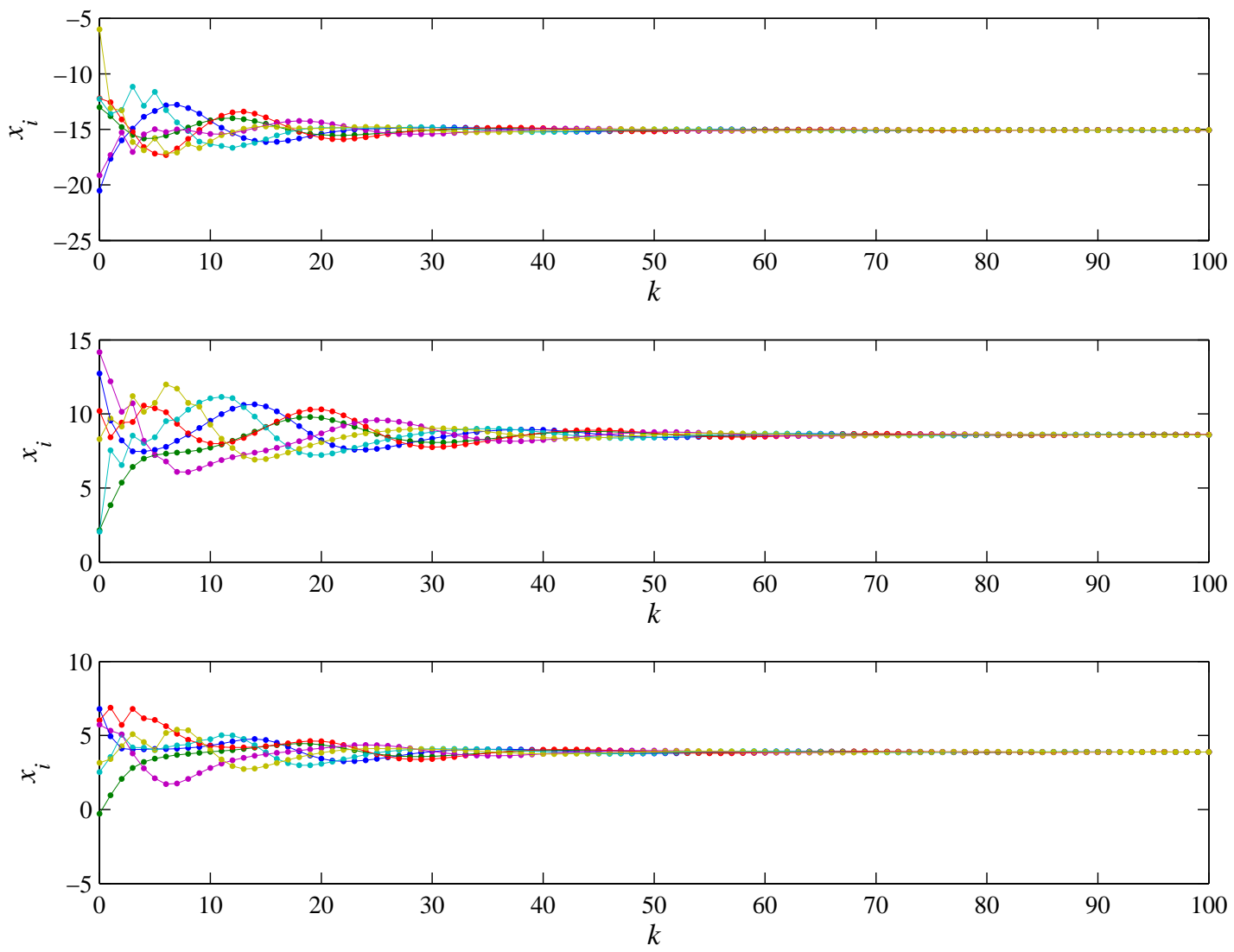

Fig. 2. The states of multi-agent system converge to the same constant.

\section{Conclusions}

We have investigated the distributed consensus problem for a class of discrete-time multi-agent network. The network under study is a directed graph and contains arbitrary distributed time delays. For consensus seeking, we developed a Lyapunov based framework and derived the theoretical results that the discretetime network with arbitrary distributed time delays reaches consensus provided that the network is strongly connected. Numerical simulation further illustrates our theoretical approach. It is possible to extend the main results to the more complicated cases such as the multi-agent systems with the time-varying interaction topology, or with the weaker connectivity that the graph contains a spanning tree, which are the future research topics.

\section{REFERENCES}

[1] P. Baldi and A. F. Atiya, "How delays affect neural dynamics and learning," IEEE Transactions on Neural Networks, vol. 5, no. 4 , pp.612-621, 1994.

[2] L. Berezansky and E. Braverman, "Oscillation of equations with an infinite distributed delay," Computers \& Mathematics with Applications Volume, vol. 60, no. 9, pp. 2583-2593, 2010.

[3] M. Cao, A. S. Morse, and B. D. O. Anderson, "Reaching a consensus in a dynamically changing environment: A graphical approach," SIAM J. Control Optim., vol. 47, no. 2, pp. 575-600, 2008. 
[4] J. Cortes and F. Bullo, "Coordination and geometric optimization via distributed dynamical systems," SIAM J. Control Optim., vol. 44, no. 5, pp. 1543-1574, 2005.

[5] L. Fang and P. Antsaklis, "Information consensus of asynchronous discrete-time multi-agent systems," in: Proc Amer Contr Conf, USA, pp. 1883-1888, 2005.

[6] M. Fiedler, "Algebraic connectivity of graphs," Czechoslovak Math. J., vol. 23, no. 98, pp. 298-305, 1973.

[7] C. Godsil and G. Royle, Algebraic Graph Theory. New York: Springer-Verlag, 2001.

[8] Y. Hatano and M. Mesbahi, "Agreement over random networks," IEEE Trans. Autom. Control, vol. 50, no. 11, pp. 1867-1872, 2005.

[9] R. A. Horn and C. R. Johnson, Matrix Analysis. Cambridge, U.K.: Cambridge Univ. Press, 1985.

[10] M. Huang and J.H. Manton, "Stochastic consensus seeking with noisy and directed inter-agent communication: fixed and randomly varying topologies," IEEE Transactions on Automatic Control, vol. 55, no. 1, pp. 235-241, 2010.

[11] A. Jadbabaie, J. Lin, and A. S. Morse, "Coordination of groups of mobile autonomous agents using nearest neighbor rules," IEEE Trans. Autom. Control, vol. 48, no. 6, pp. 988-1001, 2003.

[12] Y. Kuang, Differential Equations with Applications in Population Dynamics, Academic Press, Boston, USA, 1993.

[13] T. Li, M. Fu, L. Xie, and J. Zhang, "Distributed consensus with limited communication data rate," IEEE Transactions on Automatic Control, vol. 56, no. 2, pp. 279-292, 2011.

[14] Y. Liu, K. M. Passino, and M. M. Polycarpou, "Stability analysis of m-dimensional asynchronous swarms with a fixed communication topology," IEEE Trans. Automat. Contr., vol. 48, pp. 76-95, 2003.

[15] Y. Liu, Z. Wang, J. Liang and X. Liu, "Stability and Synchronization of Discrete-Time Markovian Jumping Neural Networks With Mixed Mode-Dependent Time Delays," IEEE Transactions on Neural Networks, vol. 20, no. 7, pp. 1102-1116, 2009.

[16] Y. Liu, Z. Wang and X. Liu, "Synchronization and State Estimation for Discrete-Time Complex Networks With Distributed Delays," IEEE Trans. SMC-B, vol. 38, no. 5, pp. 1314-1325, 2008.

[17] J. Q. Lu and D. W. C. Ho, and J. Kurths, "Consensus over directed static networks with arbitrary finite communication delays," Physical Review E, 80(6), pp. 066-121, 2009.

[18] L. Moreau, "Stability of multi-agent systems with time-dependent communication links," IEEE Trans. on Automatic Control, vol. 50, pp. 169-182, February 2005.

[19] L. Moreau and S. Belgium, "Stability of continuous-time distributed consensus algorithms," in Proc. IEEE Conf. Decision and Control, Bahamas, pp. 3998-4003, 2004.

[20] U. Münz, A. Papachristodoulou, and F. Allgöwer, "Delay Robustness in Consensus Problems," Automatica, 46(8), pp. 1252$1265,2010$.

[21] A. Okubo, "Dynamical aspects of animal grouping: swarms, schools, flocks. and herds," Adv. Biophysics, vol. 22, pp. 1-94, 1986.

[22] R. Olfati-Saber, "Distributed Kalman filter with embedded consensus filters," in Proc. 44th IEEE Conf. Decision Control Eur. Control Conf., Seville, Spain, pp. 8179-8184, Dec. 2005.

[23] R. Olfati-Saber. "Flocking for multi-agent dynamic systems: theory and algorithms," IEEE Trans. on Automatic Control, 2005, vol. 51, no. 3, pp. 401-420, 2006.

[24] R. Olfati-Saber and R. M. Murray, "Consensus problems in networks of agents with switching topology and time-delays," IEEE Trans. Autom. Control, vol. 49, pp. 1520-1533, 2004.

[25] R. Olfati-Saber, J. A. Fax, and R. M. Murray, "Consensus and cooperation in networked multi-agent systems," in Proceedings of IEEE, 95(1), Hanover, Germany, pp. 215-233, 2007.

[26] A. Papachristodoulou, A. Jadbabaie, and U. Münz, "Effects of Delay in Multi-Agent Consensus and Oscillator Synchronization," IEEE Transactions on Automatic Control, 55(6), pp.1471-1477, 2010.

[27] W. Ren and R. W. Beard, "Consensus seeking in multi-agent systems under dynamically changing interaction topologies," IEEE Trans. on Automatic Control, vol. 50, no. 5, pp. 655-661, May 2005.

[28] W. Ren and R. W. Beard, Distributed Consensus in Multi-Vehicle Cooperative Control. London, U.K.: Springer, 2008.

[29] W. Ren, R. W. Beard, and E. M. Atkins, "A survey of consensus problems in multi-agent coordination," in Proc. Amer. Contr. Conf., USA, pp. 1859-1864, Jun. 2005.

[30] C. W. Reynolds, "Flocks, herds, and schools: A distributed behavior model," Comput. Graph., vol. 21, no. 4, pp. 25-34, 1987.

[31] Y. Sun, L. Wang, and G. Xie, "Average consensus in networks of dynamic agents with switching topologies and multiple time-varying delays," Systems \& Control Letters, vol. 57, no. 2, pp. 175-183, 2008. 
[32] H. Tanner and D. Christodoulakis, "State synchronization in local-interaction networks is robust with respect to time delays," in: Proc IEEE Conference Decision and Control, Spain, pp. 4945-4950, 2005.

[33] T. Vicsek, A. Cziok, E. B. Jacob, I. Cohen, and O. Shochet, "Novel type of phase transition in a system of self-driven particles," Phys. Rev. Lett., vol. 75, no. 6, pp. 1226-1229, 1995.

[34] X. Wan, H. Fang and S. Fu, "Observer-based fault detection for networked discrete-time infinite-distributed delay systems with packet dropouts," Applied Mathematical Modelling, vol. 36, no. 1, pp. 270-278, 2012.

[35] L. Wang and F. Xiao, "A new approach to consensus problems in discrete-time multiagent systems with time-delays," Science in China Series F: Information Sciences, vol. 50, no. 4, pp. 625-635, 2007.

[36] X. Wang and G. Chen, "Synchronization in small-world dynamical networks," Int. J. Bifurc. Chaos, vol. 12, no. 1, pp. 187-192, 2002.

[37] Z. Wang, G. Wei and G. Feng, "Reliable $H_{\infty}$ control for discrete-time piecewise linear systems with infinite distributed delays," Automatica, vol. 45, no. 12, 2991-2994, pp. 2009.

[38] Z. Wu, P. Shi, H. Su, and J. Chu, "Reliable $H_{\infty}$ Control for discrete-time fuzzy systems with infinite-distributed delay, IEEE Transactions on fuzzy systems," vol. 20, no. 1, pp. 22-31, 2012.

[39] F. Xiao and L. Wang, "State consensus for multi-agent systems with switching topologies and time-varying delays," Int. J. Control, vol. 79, no. 10, pp. 1277-1284, 2006.

[40] S. Zhang, "Invariance principle for autonomous delay difference systems," Nonlinear Analysis: Theory, Methods \& Applications, Vol. 24, no. 5, pp.735-745, 1995.

[41] S. Zhang, Invariance principles for autonomous infinite delay difference systems, Northeast. Math. J. vol. 13, no. 4, pp. 411421, 1997. 